\title{
OBSERVATIONS OF THE CRAB NEBULA AT TEV ENERGIES
}

T.C.Weekes' ${ }^{1}$, C.W.Akerlof ${ }^{2}$, M.Chantell ${ }^{1}$, E.Colombo ${ }^{1}$, M.F.Cawley ${ }^{3}$, V.Connaughton ${ }^{1,4}$, D.J.Fegan ${ }^{4}$, S.Fennel1 $1^{4}$, J.Gaidos ${ }^{5}$, A.M.Hillas ${ }^{6}$, A.D.Kerrick , P.Kwok ${ }^{7}$, D.A.Lewis ${ }^{7}$, R.C.Lamb ${ }^{7}$, D.I.Meyer ${ }^{2}$, G.Mohanty ${ }^{7}$, J.Rose ${ }^{6}$, A.Rovero', G.Sembroski ${ }^{5}$, M.S.Schubnel ${ }^{2}$, M.Punch ${ }^{4}$, M.West ${ }^{6}$, T.Whitaker ${ }^{1}$, C.Wilson ${ }^{5}$.

1 Whipple Observatory, S.A.O., U.S.A.

2 University of Michigan, U.S.A.

3 st. Patrick's College, Maynooth, Ireland

4 University College, Dublin, Ireland

5 Purdue University, U.S.A.

6 University of Leeds, U.K.

7 Iowa State University, U.S.A.

\section{ABSTRACT}

The results of four years of observation of the Crab Nebula at TeV energies by the whipple group are discussed and the status of the Crab Nebula as a standard candle for $\mathrm{TeV}$ astronomy is reviewed.

\section{INTRODUCTION}

Observations with the High Resolution Camera on the $10 \mathrm{~m}$ reflector of the Whipple observatory commenced in April, 1988 . The camera and the mode of operation and analysis have been described elsewhere (Cawley et al. 1990; Vacanti et al. 1991). The results of a survey of more than 35 sources in the period 1987 to 1993 have been reported (Reynolds et al. 1993; Kerrick et al. 1993). A significant detection was found for only two sources, the Crab Nebula (Vacanti et al. 1991) and Markarian 421 (Punch et al. 1992). The former source will be discussed here; the latter is the subject of a companion paper at this symposium (Schubnell et al. 1993).

\section{CREDIBILITY}

Unlike most TeV source detections the credibility of the crab detection has increased steadily with time. The initial Whipple HRC detection was statistically significant at the 200 level using pre-determined selection criteria which were based on both the shape and orientation of the shower images (Vacanti et al. 1991). A subsequent analysis (Reynolds et al.1993) refined the selection method and increased its sensitivity by a factor of 1.75 ; this selection was then applied to three subsequent years of observation (1989-92) leading to an "a priori" detection of $31.5 \sigma$ (Punch, 1993). The actual numbers involved are shown in Table 1 . It should be noted that:

1) the signal rate is high (about 1 per minute)

2) all but $0.37 \%$ of the background has been rejected

3 ) the selected "signal" events are approximately $60 \%$ gamma rays. 


$\frac{1988-89}{\# \text { ON }}$
\#OFF
Difference
Excess $(\sigma)$
1,808 minutes
$1989-92$
\#ON
\#OFF
Difference
Excess $(\sigma)$
2,872 minutes

More convincing than the statistics of a single experiment using a new technique is the confirmation by independent experiments. This has come from four experiments using somewhat different versions of the cherenkov technique and all reporting signals at more than the $5 \sigma$ level (Table 2). It is also noteworthy that over the range from 0.2 to $10 \mathrm{TeV}$ there is no experiment with the required sensitivity that has failed to see the Crab Nebula. It would thus appear that the Crab Nebula is a good candidate for becoming the standard candle of TeV gamma-ray astronomy as it is for many other astronomies. To be thus regarded it must be clear that (a) the absolute energy and flux levels are well-established and (b) that it is truly a steady source; these considerations will be investigated in the next two sections.

Table 2 .

Ground-based Observations of the Crab Nebula

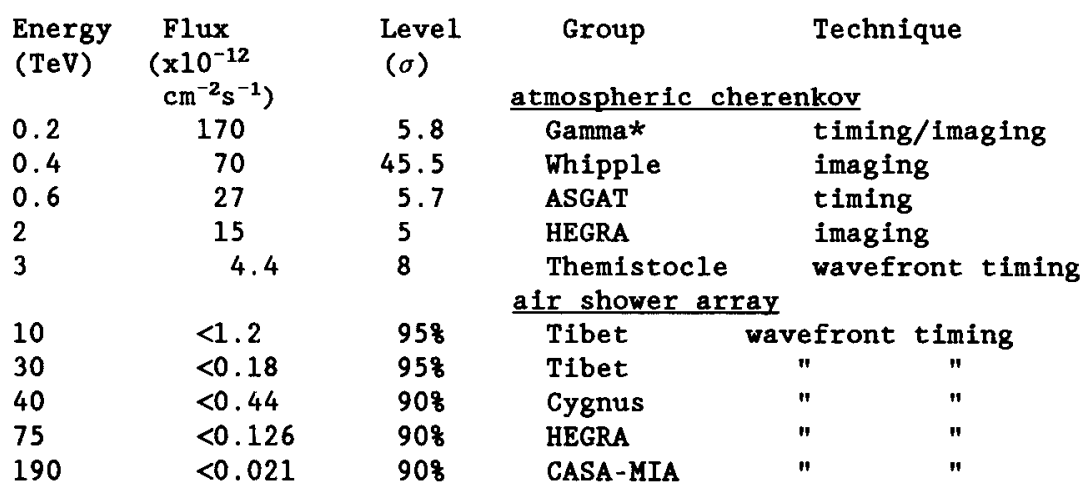

\section{SPECTRUM}

The absolute energy threshold and collection area of atmospheric Cherenkov experiments are generally not well determined which makes it difficult to construct the energy spectrum by a combination of integral points from separate experiments. The cherenkov component of air showers is 
until this conflict is resolved the utility of the crab Nebula as a TeV standard candle cannot be fully exploited.

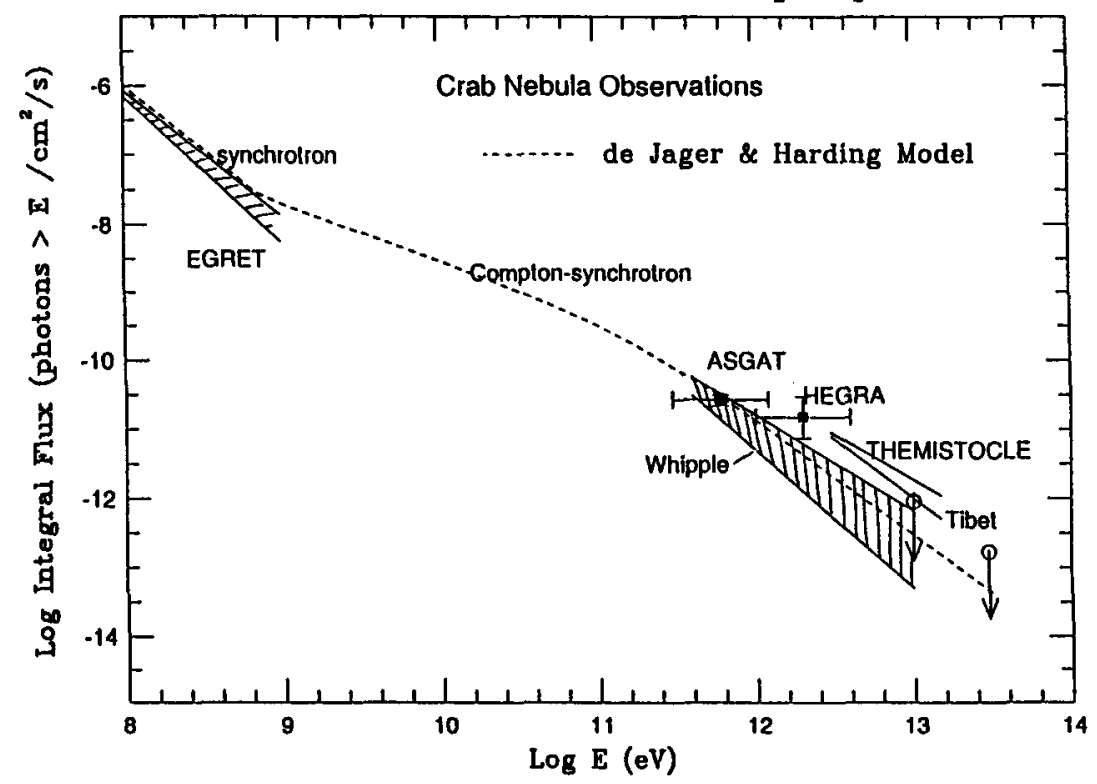

Figure 1. The Crab Nebula integral spectrum.

VARIABILITY

The first evidence for steady emission from the Crab Nebula came from a marginal detection by the first-generation detector at the Whipple observatory in 1969-72 (Fazio et al. 1973). Since the subsequent observations at TeV energies were in quantitative agreement as to flux, there was reason to hope that the source was truly constant and thus suitable as a calibration standard. Since 1988 the Crab has been detected annually with the Whipple HRC and it is now possible to look carefully for evidence for variability. This search is complicated by the fact that the threshold for the Whipple telescope was not constant due to changes in mirror reflectivity, triggering criteria and other factors. However the background response (to cosmic ray showers and to nightsky light) can be used to give an estimate of the change in sensitivity with time and hence to predict the expected gammaray rate from the crab.

In Table 3 the total counting rate as a function of season is tabulated; also shown is the Crab gamma-ray rate (using the Supercuts selection routine). To check for variability the latter must be corrected for the change in energy threshold; it is assumed that the rate scales with $\mathrm{E}^{-1.69}$. Since the gammaray collection area (using supercuts) is proportional to $\mathrm{E}^{-0.8}$, the expected gamma-ray rate must also allow for the decrease in area. 
inherently a calorimetric measure of the total shower and hence of the energy of the primary particle. Normally with a single detector it is only possible to make a density measurement; however, the atmospheric imaging technique does permit a crude measure of the shower impact parameter (based on the fact that the displacement of the shower image centroid from the optic axis i.e. the DIST parameter is a function of impact parameter on the ground). Hence showers can be selected which fall over a particular range of impact parameters where the lateral density is fairly uniform.

The first attempt to derive a differential energy spectrum from the Whipple data (selected using the Azwidth parameter) was based on Monte Carlo simulations which showed that there was a linear relationship (to the first order) between the measured light intensity and primary gamma-ray energy for energies between 0.4 and $4 \mathrm{TeV}$ for impact parameters between $50 \mathrm{~m}$ and $125 \mathrm{~m}$ (Vacanti et al. 1991). The resulting spectrum was:

$$
\frac{\mathrm{dN}(\mathrm{E})}{\mathrm{dE}}=(2.5) \times 10^{-11}(\mathrm{E} / 0.4 \mathrm{TeV})^{-(2.4 \pm 0.1 \pm 0.3)} \text { photons } \mathrm{m}^{-2} \mathrm{~s}^{-1} \mathrm{TeV}^{-1}
$$

where the quoted errors are, respectively, the $1 \sigma$ statistical errors and the estimated systematic errors.

More detailed simulations indicate that the light intensity-primary energy relationship is more complex but that the energy spectrum can be unfolded (Lewis et al. 1993). First, the gamma-ray selection criteria are modified so that the telescope collection area is large and relatively independent of energy, This is achieved by using selection criteria that are energy dependent; this is achieved at some loss in the overall significance. Second, a method is established for estimating the gamma-ray energy and finding the corresponding energy resolution function. The fractional energy resolution $(\mathrm{dE} / \mathrm{E})$ is about $40 \%$.

The best power-law fit for the differential spectrum is:

$$
\frac{\mathrm{dN}(\mathrm{E})}{\mathrm{dE}}=(1.48 \pm 0.09 \pm 0.41) \times 10^{-7}(\mathrm{E} / \mathrm{TeV})^{-(2.69 \pm 0.09 \pm 0.3)} \text { photons } \mathrm{m}^{-2} \mathrm{~s}^{-1} \mathrm{TeV}^{-1}
$$

The systematic errors arise from the uncertainties in the estimate of the energy threshold which, in turn, arise largely from uncertainties in the overall mirror reflectivity at the time the measurements were made; by allowing a $\pm 25 \%$ change in the assumed reflectivity the systematic error is estimated. The resulting spectrum (with the estimated uncertainties) is plotted in Figure 1 and compared with the predicted spectrum of the synchrotron-Compton model (De Jager and Harding, 1992). From the figure one can see some scatter in absolute flux values. For the two groups that have measured a spectrum there is agreement on the spectral index ( - -1.6 integral). Unfortunately, they disagree on the flux by a value of 3 , and 


$\begin{array}{llccc}\text { Observed and Expected crab Rates } \\ \text { Season } & \begin{array}{l}\text { Energy } \\ (\mathrm{TeV})\end{array} & \begin{array}{l}\text { Background } \\ \left(\mathrm{min}^{-1}\right)\end{array} & \begin{array}{l}\text { Observed } \\ \left(\mathrm{min}^{-1}\right)\end{array} & \begin{array}{c}\text { Predicted } \\ \left(\mathrm{min}^{-1}\right)\end{array} \\ 1988-89 & 0.40 & 273 & 1.49 & \\ 1989-90 & 0.44 & 229 & 1.13 & 1.14 \\ 1990-91 & 0.52 & 176 & 0.77 & 0.77 \\ 1991-92 & 0.56 & 155 & 0.55 & 0.65\end{array}$

The apparent drop in 1991-2 is not statistically significant. Thus there is good evidence that the flux was constant within the experimental limits over the four year period 1988-92. It should be noted that at this symposium it was reported that between 1991 and 1992 there was a decrease noted in the steady MeV-GeV Crab flux detected by EGRET (De Jager et al. 1993). On the De Jager-Harding model the gammaray flux at EGRET energies would come from the synchrotron radiation of electrons close to the pulsar whereas the GeV-TeV gamma rays would come from the compton scattering of photons throughout the nebula; the latter would not be expected to be variable on short times-scales.

\section{CONCLUSION}

The existence of the Crab Nebula as an unpulsed TeV source now rests on very strong ground; while there is still some disagreement in the absolute flux values there is no question that this is the best available standard candle for the calibration of $\mathrm{TeV}$ telescopes. Prior to the launch of GRO it was predicted from $\mathrm{TeV}$ observations that there would be a flattening of Crab Nebula spectrum at energies above $10 \mathrm{GeV}$ (Weekes et al. 1989) and a steepening of the pulsed component; this is apparently now confirmed by EGRET (Nolan et al. 1993).

Acknowledgement. This work has been supported by the U.S.Department of Energy, NASA, and Eolas (Ireland).

\section{REFERENCES}

Cawley, M.F. et al., 1991, Experimental Astronomy 1, 185. Clear, J. et al., 1987, Astron. and Astrophys., 174, 85. De Jager, O.C. and Harding, A.K., 1992, Ap. J., 396, 161. Fazio, G.G. et al., 1972, Ap. J. Lettr. 175, L117.

De Jager, 0 . et al. 1993, (this symposium).

Kerrick, A. et al. 1993, Proc. 23rd ICRC (Calgary), 1, 405.

Lewis, D.A. et al. 1993, Proc. 23rd ICRC (Calgary), 1, 279.

Nolan, P.L. et al. 1993, Ap. J. $409,697$.

Punch, M., 1993, Ph.D.Dissertation, N.U.I. (Unpublished)

Punch, M. et al., 1992, Nature, 358, 477.

Reynolds, P.T. et al., 1993, Ap.J., 404, 218.

Schubnell, M. et al. 1993, Proc. 23rd ICRC (Calgary), 1, 409.

Vacanti et al., 1991, Ap.J. 377, 467.

Weekes, T.C. et al., 1989, Ap. J. $342,379$. 